Trends Endocrinol Metab. 2013 July ; 24(7): 332-341. doi:10.1016/j.tem.2013.03.001.

\title{
A fatty gut feeling
}

\author{
Daniele Piomelli ${ }^{1,2}$ \\ ${ }^{1}$ Departments of Anatomy and Neurobiology, Pharmacology, and Biological Chemistry, University \\ of California Irvine, CA 92612, USA \\ ${ }^{2}$ Department of Drug Discovery and Development, Istituto Italiano di Tecnologia, Genoa 16163, \\ Italy
}

\section{Abstract}

The absorptive epithelium of the proximal small intestine converts oleic acid released during fat digestion into oleoylethanolamide (OEA), an endogenous high-affinity agonist of peroxisome proliferator-activated receptor-a (PPAR-a). OEA interacts with this receptor to cause a state of satiety characterized by prolonged inter-meal intervals and reduced feeding frequency. The two main branches of the autonomic nervous system, sympathetic and parasympathetic, contribute to this effect: the former by enabling OEA mobilization in the gut and the latter by relaying the OEA signal to brain structures, such as the hypothalamus, that are involved in feeding regulation. OEA signaling may be a key component of the physiological system devoted to the monitoring of dietary fat intake, and its dysfunction might contribute to overweight and obesity.

\section{A multifunctional fat sensor}

The ability to monitor the nutrient composition of food allows animals to generate neural and endocrine feedback signals that adapt behavior and metabolism to environmental fluctuations in food availability. Being able to sense dietary fat is especially important, from an adaptive perspective, because of the high energydensity of this nutrient and the essential role of lipids as building blocks of cell membranes and precursors for hormones and other bioactive molecules. The need to secure appropriate amounts of fat and optimize its storage and utilization provides a plausible evolutionary basis for the existence of multiple lipidsensing mechanisms throughout the mammalian body. In the mouth, the first relay station of this diffused monitoring system, dietary fat triggers a potent stimulatory effect on feeding [1]. Experiments in rodents have shown that the presence of this nutrient in the oral cavity is sufficient to activate taste-responsive neurons in the nucleus of the solitary tract (NST; see Glossary) [2], stimulate subcortical regions of the brain involved in the control of rewardoriented behavior [3], and cause release of endocannabinoids in the proximal gut [4]. These events are considered to be important in determining the innate attraction to high-fat foods experienced by mammals $[1,5]$ and are likely mediated by molecular sensors found on taste buds. Among the sensors identified so far are the membrane protein CD36 [6-8], the G protein-coupled receptors GPR120 and GPR40 [9,10], and the calcium-activated cation channel TRPM5 (transient receptor potential cation channel M5) [11,12].

In addition to promoting food intake through the cephalic mechanisms mentioned above, dietary fat also exerts profound satiety-inducing effects that result from the recruitment of biosensors localized in the upper portions of the small intestine - in other words the duodenum and jejunum. The infusion of lipid emulsions into the duodenum markedly

(C) 2013 Elsevier Ltd. All rights reserved.

Corresponding author: Piomelli, D. piomelli@uci.edu. 
suppresses food intake in both rodents and humans [1,13]. In rats, this satiating action is abrogated by removal of the afferent nerves that connect the gastrointestinal tract to the brain [14], and is accompanied by activation of vagal afferents in the gut [15] and neurons in the NST [16]. The local release of two well-known gut hormones, cholecystokinin and serotonin, has been implicated in these responses [17]. New evidence suggests, however, an important role for a different class of signaling molecules - the amides of fatty acids (FAs) with ethanolamine (fatty acid ethanolamides, FAEs) (Figure 1).

The FAEs were first isolated from plant and animal tissues more than 50 years ago $[18,19]$ and were found to possess strong anti-inflammatory activity [20]. However, these substances received little attention until the discovery of anandamide, a polyunsaturated FAE that binds to and activates $G$ protein-coupled cannabinoid receptors [21]. The recognition of the significance of anandamide as an endocannabinoid transmitter [22] fueled a renewed interest in other members of the FAE family and led to uncovering the function served by these molecules as regulators of food intake [23] and, eventually, to identifying PPAR- $\alpha$ as a key cellular effector of this regulatory action [24]. It also became apparent that the FAEs participate in a variety of physiological and pathological processes in addition to feeding including pain [25-27], innate immunity [28,29], and reward regulation [30] - and may thus represent a previously unrecognized class of multifunctional lipid mediators (Box 1).

\section{Box 1}

\section{An ancient class of protective signals}

Particular classes of chemicals, such as cyclic nucleotides and biogenic amines, seem to have been especially tagged by evolution to serve as cellular signals. The FAEs are one such class. Found throughout the phylogenetic tree, from slime molds to humans, these lipid molecules play an important role in the protective response mounted by flowering plants against harmful organisms. Exposure to xylanase, a pathogen produced by parasitic fungi, causes cells of the tobacco plant (Nicotiana tabacum) to release into the extracellular medium lauroylethanolamide and myristoylethanolamide, two saturated FAEs that stimulate expression of the defense gene $P A L 2$ and attenuate the cellular changes evoked by xylanase [85]. In addition to effecting innate immune reactions, FAEs may also contribute to the control of plant germination, as suggested by the profound changes in seed FAE levels observed during sprouting and by the ability of exogenous FAEs to inhibit this process [85]. Although the molecular targets for FAEs in plants are still unknown, various receptors have been identified for these substances in animals including the $\mathrm{CB}_{1}$ and $\mathrm{CB}_{2}$ cannabinoid receptors, GPR119, TRPV1, and PPAR-a (see main text). In mammalian cells, saturated and monounsaturated FAEs, such as PEA and OEA, exert marked cytoprotective effects that are mediated by PPAR-a. Activation of this nuclear receptor underpins the ability of PEA and OEA to attenuate peripheral inflammation [28,29] and neuronal toxicity [86]. For example, studies in rodents indicate that lipopolysaccharide (LPS), a chemical constituent of the bacterial cell wall, evokes a rapid downregulation of NAPE-PLD expression in a macrophage cell line; this requires Toll-like receptor activation and results in a decrease in intracellular PEA and OEA levels [87]. Pharmacological blockade of NAAA activity normalizes such levels and inhibits LPS-induced inflammation through a PPAR-a-dependent mechanism $[29,87]$. Tonic FAE production in macrophages may thus provide a no-go signal that prevents the unleashing of inappropriate responses to potentially harmful stimuli - an intriguing evolutionary avatar of the defensive actions exerted by FAEs in plants.

The present article reviews current data suggesting that OEA (Figure 1) and a subset of structurally similar FAEs are key components of the molecular machinery responsible for 
the monitoring of fat intake in the small intestine. After an outline of the enzyme pathway involved in the formation and degradation of OEA, the review describes mechanisms mediating the mobilization of these lipid substances in response to fat, their effects on feeding behavior, and the role of PPAR-a and other receptors in such effects. Considerations on the physiological significance of OEA signaling conclude this overview.

\section{OEA formation and deactivation}

The biochemical pathway thought to be responsible for the production and degradation of OEA in mammalian cells is illustrated in Figure 2. Its first committed step is the exchange of FA between two membrane phospholipids, with an acyl group being transferred from the stereospecific numbering-1 ( $s n-1)$ position of phosphatidylcholine to the amine group of phosphatidylethanolamine (PE). This reaction is catalyzed by a calcium-dependent enzyme activity (referred to as $N$-acyltransferase), which remains to be molecularly identified, and yields a chemically heterogeneous family of $\mathrm{N}$-acylphosphatidylethanolamine (NAPE) species [31,32]. Members of this family differ in the fatty acyl moiety linked to three distinct points of the PE scaffold: (i) the $s n-1$ position, which usually contains a saturated FA; (ii) the $s n-2$ position, which incorporates an unsaturated or polyunsaturated FA; and (iii) the amine group of ethanolamine, which can bind FAs with various numbers of double bonds, and determines the identity of the FAE produced in the subsequent enzymatic step (see below). Another element of heterogeneity among NAPEs is represented by the optional replacement of the $s n-1$ acyl ester group with an alkyl or alkenyl ether moiety. These $s n-1$ ether NAPEs (also known as NAPE plasmalogens) do not appear to be interchangeable with their $s n-1$ acyl ester counterparts. For example, the mucosal layer of the rat intestine contains almost exclusively $s n-1$ acyl ester NAPEs, whereas the rest of the gut wall and the brain are enriched in $s n-1$ alkyl and alkenyl species $[33,34]$. The functional significance of this anatomical segregation, if any, is still unknown.

NAPE species that incorporate oleic acid at the amine position generate OEA upon hydrolysis of their distal phosphodiester bond (Figure 2) [31,32]. This reaction is catalyzed by a NAPE-selective phospholipase D (NAPE-PLD), which was purified and molecularly cloned in the laboratory of Natsuo Ueda [35]. NAPE-PLD cleaves different members of the NAPE family with similar efficiency and is broadly expressed in mammalian tissues [35]. In the mouse duodenum - where NAPE-PLD is found in the enterocyte layer as well as the underlying lamina propria - feeding enhances both the activity and expression of this enzyme [33]. Notably, however, targeted disruption of the NAPE-PLD gene (Napepld) in mice does not completely suppress OEA production, which is suggestive of a complementation by redundant biosynthetic routes [36,37].

The hydrolysis of OEA into oleic acid and ethanolamine is considered to be the primary mechanism through which the biological actions of this lipid mediator are terminated (Figure 2). Two intracellular amidases have been implicated in this process: FA amide hydrolase (FAAH) [38] and $N$-acylethanolamine acid amidase (NAAA) [39]. FAAH is a member of the amidase signature family of serine hydro-lases [38] and is highly expressed in the central nervous system (CNS), liver, and small intestine [40]. Electron microscopy studies in rat brain tissue have shown that this integral membrane protein is associated with cytoplasmic organelles such as the endoplasmic reticulum and mitochondria [41]. Similarly to NAPE-PLD, FAAH is found in epithelial and lamina propria cells of the mouse jejunum but, in contrast to NAPE-PLD, food intake lowers its local activity and expression levels rather than increasing them [33]. There are two known isoforms of FAAH, FAAH-1 and FAAH-2, the latter being exclusively present in humans and other placental mammals [42]. A second OEA-hydro-lyzing enzyme, NAAA, belongs to the choloylglycine family of cysteine hydrolases and shares no sequence homology with FAAH [39], from which it also 
differs for its highly localized expression in macrophages [43] and preference for palmitoylethanolamide (PEA) as a substrate [39]. Pharmacological evidence suggests that NAAA regulates tissue FAE levels during inflammation [27,29], but its role in smallintestinal OEA degradation remains to be defined.

\section{Diet-derived oleic acid triggers OEA mobilization in the gut}

Feeding promotes OEA formation in the small intestine of various vertebrate species, including rats [23,44], mice [33], and Burmese pythons [45]. Studies in rats suggest that three simultaneous biochemical events are responsible for this effect: (i) enhanced production of NAPEs that contain oleic acid at the amine position (i.e., OEA precursors); (ii) activation of NAPE-PLD, which accelerates the release of OEA from NAPEs; and (iii) inhibition of FAAH activity, which favors the accumulation of newly formed OEA [33]. Despite the diversity of NAPE species present in the gut, food intake selectively induces the formation of OEA and its close analog, linoleoylethanolamide (LEA), without affecting the levels of other equally abundant FAEs, such as PEA and stearoylethanolamide [33]. This suggests that NAPE generation is the initial rate-limiting step in food-induced OEA mobilization, which drives the subsequent cleavage of NAPEs by NAPE-PLD (Figure 2). It is important to point out that feeding mobilizes OEA only in the luminal layer of the small intestine. In fact, food ingestion does not change OEA levels in the gut submucosal layers (submucosa, muscularis propria, and serosa), stomach, or colon, and actually lowers them in blood and liver, through a mechanism that remains unknown [33].

Experiments in which individual nutrients were infused separately into the duodenum of catheterized rats showed that fat $\left(\right.$ Intralipid $\left.^{\circledR}\right)$ is a potent stimulus for jejunal OEA production, whereas sugar (glucose) and protein $\left(\right.$ Peptone $^{\circledR}$ ) have no such effect [46]. More detailed investigations into the identity of the lipid substance(s) involved in this response revealed that intraduodenal infusions of oleic acid, but not palmitic acid, recapitulate the coordinated regulatory effect elicited by food intake on small-intestinal OEA metabolism in that they enhance the biosynthesis of OEA-releasing NAPEs and regulate the activities of NAPE-PLD and FAAH, resulting in an overall increase in OEA levels. These findings indicate that the presence of non-esterified ('free') oleic acid in the lumen of the small intestine stimulates mucosal cells to generate OEA [46].

Free oleic acid is generated in the gut lumen during digestive breakdown of complex dietary lipids. How do gut cells detect the released fatty acid? Studies with genetically modified mice revealed that deletion of CD36 abrogates food-induced OEA production, suggesting that this protein acts as a biosensor for food-derived oleic acid $[46,47]$. Other known attributes of CD36 are consistent with such a role, including: (i) its presence on the apical surface of enter-ocytes in rodent duodenum and jejunum [7], (ii) its function as a taste receptor for unsaturated FAs in the mouse oral cavity $[8,48,6]$, (iii) its association with Src family kinases involved in intracellular signal transduction [49], (iv) its possible contribution to the satiety induced by a high-protein diet [50], and (v) its participation in the transport of unsaturated FAs across the plasma membrane of enter-ocytes in the upper gut [51]. The latter property suggests that CD36 might contribute to OEA mobilization by facilitating, either directly or indirectly, the mucosal uptake of oleic acid. This idea was tested by infusing into the duodenum of mice the chemical tracer, 10Z-heptadecenoic acid, which closely resembles oleic acid but is not normally present in mammalian tissues. As the hypothesis predicted, the tracer was efficiently taken up by mucosal cells of wild type mice, but not by cells of mice lacking CD36, and was then converted into its corresponding NAPE ( $N$-10Z-heptadecenoyl-PE) and FAE (10Z-heptadecenoylethanola-mide) [46]. 
Figure 3 illustrates a possible mechanism through which fat-containing foods might trigger OEA biosynthesis in the small intestine. According to this model, enter-ocytes lining the lumen of the proximal gut capture oleic acid derived from the digestion of dietary fat, internalize the fatty acid, and then direct it toward the production of NAPE and OEA. CD36 plays an obligatory role in this process, but its precise functions are not entirely clear. In addition to promoting oleic acid uptake, as discussed above, CD36 might also regulate the activities of NAPE-PLD and FAAH, possibly by engaging Src-family protein tyrosine kinases such as Fyn or Syk, which are known effectors of CD36-dependent signaling in platelets [49]. Although various aspects of this lipid-sensing process remain speculative, there is evidence that excessive fat intake may render the process dysfunctional. Harald Hansen and his collaborators have reported that prolonged exposure to dietary fat reduces small-intestinal FAE levels in rats [52,53], raising the possibility that diets rich in fat might promote overeating, at least in part, by suppressing the satiating effects of gut-derived OEA. It is to such effects that we must now turn our attention.

\section{OEA produces satiety}

Administration of OEA through the intraperitoneal (IP) or oral route causes a long-lasting inhibition of food intake in rats and mice [23,24,54-58]. This response is structurally selective in that close analogs of OEA are either inactive (e.g., oleic acid) or less potent than OEA (e.g., LEA and PEA) when administered to rats by IP injection [23,53]. Given the diversity of factors that can influence feeding in a nonspecific manner, it is important to point out that OEA does not produce behaviors that are indicative of fear or anxiety, does not change plasma corticosterone levels, and does not induce conditioned taste aversion in rats - indicating that its hypophagic actions cannot be attributed to stress or malaise [23,57]. The behavioral selectivity of OEA is further underscored by its lack of effect on water intake and sodium appetite [23,57].

Rats consume most of their food during the night, in a series of meals that are separated from one another by time intervals of variable duration. Anorexic agents modify distinct aspects of this patterned behavior. For example, the gut peptide cholecystokinin selectively reduces meal size [59], whereas the serotonergic agonist $D$-fenfluramine shortens meal duration and prolongs the time preceding the first nighttime meal [60]. The hypophagic actions of OEA are different from those of cholecystokinin or $D$-fenfluramine, and appear to depend on the feeding state of the animal. In free-feeding rats, OEA decreases meal frequency without altering meal size; by contrast, the compound simultaneously reduces these two parameters in food-deprived animals [54].

The data summarized above document the ability of OEA to modulate feeding, when administered as a drug, and raise the question as to whether endogenous OEA might exert similar effects. This possibility was addressed by injecting a viral vector directing overexpression of NAPE-PLD into the duodenum of rats, and monitoring OEA formation and feeding behavior during the following 12 days [61]. The expected accrual in jejunal NAPE-PLD transcription, which reached its peak 8-10 days after virus injection, was accompanied by a local increase in OEA content and a concomitant decrease in food intake. The latter response resembled that evoked by exogenous OEA in that it resulted from a prolongation of feeding latency and inter-meal interval, rather than from a reduction in meal size [61]. A plausible interpretation of these findings is that elevations in OEA levels that are spatially restricted to the wall of the upper gut are sufficient to modulate food intake in rats. 


\section{PPAR- $\alpha$ mediates the satiety-inducing effects of OEA}

Several lines of evidence support the idea that OEA causes satiety by activating PPAR-a, a nuclear receptor that is also implicated in the mechanism that allows liver cells to gauge the levels of circulating FAs [62,63]. The available evidence can be summarized as follows: (i) OEA binds to the purified ligand-binding domain of PPAR- $a$ with a $K_{\mathrm{D}}$ of $\sim 40 \mathrm{nM}$ and stimulates the transcriptional activity of this protein, in cell-based assays, with a median effective concentration $\left(\mathrm{EC}_{50}\right)$ of $\sim 120 \mathrm{nM}$ [24]; (ii) the latter effect is selective for OEA because close structural analogs of this compound are either inactive (e.g., stearoylethanolamide) or less active than OEA (e.g., PEA; $\mathrm{EC}_{50} \sim 3 \mu \mathrm{M}$ ) [24,28]; (iii) OEA does not inhibit food intake in mice lacking PPAR-a, which respond normally to other anorexic agents [24]; (iv) the hypophagic actions of OEA are mimicked by two chemically distinct PPAR- $\alpha$ agonists (GW-7647 and Wy-14643), but not by agonists of other PPAR subtypes (PPAR- $\gamma$ and PPAR- $\delta$ ) [24,58]; (v) OEA stimulates the transcription of various PPAR- $\alpha$ target genes in small-intestinal mucosa and liver - including PPAR- $\alpha$ itself, CD36, and tissue-specific fatty acid-binding proteins [24]; (vi) after feeding, the concentrations of endogenous OEA in gut mucosa rise to levels $(\sim 300 \mathrm{nM})$ that are sufficient to activate fully PPAR-a (EC $50 \sim 120 \mathrm{nM}$ ) [33]; and, finally, (vii) targeted disruption of the PPAR-a gene (Ppara) in mice abrogates the satiety response induced by fat and increases feeding frequency, without changing other aspects of ingestive behavior such as food probing or eating rate [46].

One argument against the identification of PPAR- $\alpha$ as a receptor for OEA is that this compound acts within minutes of its administration, whereas the transcriptional changes set in motion by PPAR-a unfold over a period of hours. This discrepancy is only apparent, however, because emerging evidence indicates that PPAR- $\alpha$ exerts its manifold cellular effects by engaging both transcriptional and non-transcriptional mechanisms. For example, Virginia Seybold and her collaborators have shown that two structurally distinct PPAR-a agonists, GW-7647 and PEA, cause a rapid decrease in the amplitude of evoked $\mathrm{Ca}^{2+}$ transients in mouse sensory neurons when these are cultured together with tumor cells, and that this response is blocked by a PPAR- $\alpha$ antagonist [64]. Similarly, Miriam Melis and Marco Pistis reported that PPAR-a activity modulates the firing rate of neurons in the rat midbrain through a fast effect on nicotinic receptors [65]. These and other electrophysiological studies [26] demonstrate that PPAR- $\alpha$ can influence membrane ionchannel activities by recruiting intracellular signal transduction pathways that are independent of gene expression.

In addition to the nuclear receptor PPAR- $\alpha$, two cell-surface proteins have been also implicated in the biological actions of OEA. Experiments in Xenopus oocytes have shown that OEA activates the capsaicin receptor TRPV1 (transient receptor potential cation channel vanilloid-1), but only when this receptor has been previously phosphorylated by protein kinase C $[66,67]$. Full concentration-response curves for this effect have not been reported. However, studies in mouse vagal sensory neurons indicate that OEA $(10 \mu \mathrm{M})$ evokes an inward TRPV1-mediated current that is approximately $20 \%$ the size of the current elicited by a maximally active concentration of capsaicin $(0.25 \mu \mathrm{M})$ [67]. In agreement with the established role of TRPV1 in the processing of painful stimuli, IP or intradermal injections of OEA evoke short-lasting nociceptive responses in wild type mice, but not in mice lacking TPRV1 [26,67]. OEA also acts as a medium-potency agonist for GPR119 $\left(\mathrm{EC}_{50} \sim 3 \mu \mathrm{M}\right)$ [68], a $\mathrm{G}$ protein-coupled receptor that recognizes a broad panel of lipid molecules in addition to OEA [69]. GPR119 is expressed in intestinal endocrine L-cells, which secrete glucagon-like peptide-1 (GLP-1), and intraluminal infusions of OEA were found to increase circulating GLP-1 levels in rats [70]. Nevertheless, the observation that genetic deletion of 
TRPV1 or GPR119 in mice does not alter the anorexic effects of OEA strongly argues against a direct involvement of these receptors in OEA-induced satiety [71,72].

\section{From gut to brain}

Peripheral cholecystokinin regulates food intake by activating vagal sensory fibers that travel from the small-intestinal epithelium to the brainstem NST [59,73] (Figure 4). The fact that dietary fat elevates OEA levels only in the upper gut suggests that this lipid mediator might also produce its anorexic effects through a mechanism mediated by the vagus nerve (Box 2). This idea finds support in the following observations: (i) OEA reduces food intake after systemic administration, but not after infusion into the brain ventricles [23]; (ii) local elevations in small-intestinal OEA production (such as those resulting from food ingestion or NAPE-PLD overexpression) mimic the hypophagic effects of exogenous OEA [61]; (iii) surgical disconnection of the vagus nerve prevents such effects, but not those of centrally acting anorexiants [23]; (iv) a similar failure to respond to OEA is seen in rats treated with neurotoxic doses of capsaicin, which deprive the animals of peripheral vagal and non-vagal sensory fibers [23]; and, finally (v) IP injections of OEA stimulate transcription of c-Fos (a marker of neuronal activation) in the NST [23,74]. Collectively, the evidence summarized above suggests that enterocyte-derived OEA inhibits food intake by recruiting sensory afferents in the intestinal branch of the vagus nerve (Figure 4). However, the exact mechanism underlying this effect remains unknown,

\section{Box 2}

\section{NAPE as a circulating satiety hormone}

Consuming a fatty meal does not increase the levels of OEA in blood - it actually decreases them - arguing against a role for this lipid substance as a circulating hormone [33]. Evidence indicates, however, that another gut-derived lipid substance might serve such a function. Gerald Shulman and his collaborators reported in 2008 that feeding rats a high-fat diet or infusing a lipid emulsion into their duodenum caused a rise in lymph and plasma NAPE concentrations [88]. Chemical analyses revealed that the predominant NAPE species in plasma affected by fat feeding were those incorporating palmitic or stearic acid at the amine position of the phosphatidy-lethanolamine (PE) scaffold, which are not precursors for OEA (see Figure 2 in main text). When one of these NAPEs was administered systemically in rats it reduced food intake without causing conditioned taste aversion. This anorexic effect did not require NAPE hydrolysis and was readily distinguishable from that elicited by OEA in that it persisted following resection of the vagus nerve and was reproduced by infusion of NAPE into the brain lateral ventricle. The results suggest that gut-derived NAPEs are released into the circulation and regulate food intake through a centrally mediated mechanism [88]. This idea was challenged by recent collaborative experiments conducted in the laboratories of Harald Hansen and Natsuo Ueda, who showed that: (i) the high IP dosages of NAPE required for feeding inhibition (100-1000 mg. $\mathrm{kg}^{-1}, 20$-fold higher than for OEA) caused substantial reductions in motor activity, and (ii) two lipid substances structurally different from NAPE, PE, and phosphatidic acid (PA), decreased food intake when administered in the same dose range as NAPEs [89]. Although suggestive of a nonspecific hypophagic effect, these findings should be interpreted with caution. For example, the observation that NAPEs reduce food intake more effectively in leptin-deficient than they do in wild type mice argues in favor of a selective mechanism of action [88]. Additional studies are needed to resolve this uncertainty. 


\section{Neural control}

Anthony Sclafani and his collaborators reported that surgical disconnection of the celiacsuperior mesenteric ganglion (CSMG) complex - the sympathetic node that sends noradrenergic projections to the intestine and other visceral organs - impairs the ability of intraduodenal fat infusions to reduce food intake [14]. Subsequent investigations demonstrated that resection of the CSMG complex in rats shortens the interval between meals - a response opposite to that evoked by OEA - and prevents food-induced OEA production in the gut [75]. The finding that pharmacological antagonists of $\beta_{2}$-adrenergic receptors also interrupt small-intestinal OEA mobilization provides further support for an enabling role of post-ganglionic sympathetic fibers in gut OEA signaling [75] (Box 3).

Because such fibers are anatomically segregated to the deeper layers of the gut (submucosal layer and myenteric plexus) [76], it seems likely that diffusible factors and/or enteric neural networks transmit the sympathetic signal to the mucosal epithelium, where the bulk of foodstimulated OEA biosynthesis takes place (Figure 4).

\section{Box 3}

\section{OEA signaling in adipose cells}

The sympathetic nervous system enables fat-induced OEA signaling in the gut, but does not directly stimulate it [75]. By contrast, sympathetic activity alone is sufficient to trigger OEA formation in white adipose tissue. Keeping rats in a cold room $\left(4^{\circ} \mathrm{C}\right)$ for $6 \mathrm{~h}$ increased OEA biosynthesis in their fat pads, but not liver or intestine [90]. This effect was prevented by administration of the $\beta$-adrenergic antagonist propranolol and was reproduced, in vitro, by incubating isolated adipose cells with a $\beta$-adrenergic agonist [90]. Sympathetic activation is a canonical response to cold exposure, and one of its key biochemical consequences is the mobilization of free fatty acids from adipose triacylglycerol stores: norepinephrine secreted from sympathetic varicosities binds to $\beta$ adrenergic receptors on the surface of adipose cells, which stimulate hormone-sensitive lipase activity via cAMP-dependent protein phosphorylation. Does OEA participate in this important physiological process? Working with freshly dissociated rat and mouse adipocytes, Manuel Guzman and collaborators found that incubation with exogenous OEA increased the release of non-esterified fatty acids and glycerol into the extracellular medium with an $\mathrm{EC}_{50}$ of $\sim 2 \mu \mathrm{M}$ [91]. This lipolytic effect required PPAR-a, because it did not occur in cells prepared from PPAR-a-deficient mice, but was independent of changes in intracellular cAMP. Importantly, substantial fatty acid mobilization occurred also, in vivo, 60-120 min after IP administration of OEA to lean rats (median effective dose, $\mathrm{ED}_{50} \sim 2.6 \mathrm{mg} \cdot \mathrm{kg}^{-1}$ ). OEA did not change the plasma levels of glucose, insulin, or glucagon, but markedly increased the transcription of several adipose-tissue genes involved in lipid transport, including CD36 and fatty acid-binding protein [91]. Similar results were obtained when OEA was administered chronically (for 2 weeks or more) in rats or mice rendered obese by exposure to a high-fat diet [91-93]. Although still incomplete, the available data allow us to hypothesize that sympathetic activity controls OEA release in adipose tissue, where this lipid mediator might act as an autocrine regulator of lipid transport function.

In addition to the NST, IP OEA injections stimulate c-Fos expression in peptide-secreting neurons of the para-ventricular (PVN) and supraoptic (SON) nuclei of the hypothalamus $[23,74]$. Studies in rats have shown that systemic OEA administration causes an increase in the expression of the neuropeptide oxytocin, but not vasopressin, in magnocellular neurons of the PVN and SON [74]. Moreover, pharmacological blockade of central oxytocin receptors abrogates the hypophagic effects of OEA, implying that release of oxytocin in the 
hypothalamus and/or other regions of the brain may be a key effector of OEA-induced satiety [74]. The identification of a functional link between OEA signaling in the gut and oxytocin transmission in the CNS raises the intriguing, but as-yet unexplored possibility, that OEA might also trigger other actions of oxytocin, such as facilitation of social behaviors [77].

Noradrenergic projections that connect the NST to the basolateral complex of the amygdala (BLA) are part of a neural circuit that is crucially implicated in the consolidation of recent emotional memories [78]. Considering that the ability to retain contextual information associated with nutrient sources would provide an adaptive advantage to animals foraging in the wild, it is plausible that OEA might reinforce such ability by strengthening memory consolidation [79]. Using two distinct experimental paradigms in rats - inhibitory avoidance and the Morris watermaze -Campolongo et al. found that IP administration of OEA after behavioral training strongly improved the retention of these tasks. This effect was blocked by infusions of the local anesthetic lidocaine into the NST or the $\beta$-adrenergic antagonist propranolol into the BLA, indicating that the signal launched by peripheral OEA gains access to the CNS via the afferent vagus, and strengthens memory consolidation by stimulating noradrenergic activity in the BLA [79] (Figure 4). It appears, therefore, that OEA signaling in the gut initiates an integrated response in which satiety induced by a fatrich meal coincides temporally with enhanced encoding of information about the spatial and emotional context in which the meal was consumed.

\section{Regulation of fat absorption and gastrointestinal motility}

We have seen in a preceding section that OEA stimulates small-intestinal mucosal cells to express various PPAR-a target genes involved in the transport of dietary lipids, including CD36 and intestinal fatty acid-binding protein [24]. The possibility that OEA-mediated signaling might contribute to the control of fat absorption was confirmed in an experiment that assessed the effects of this compound on FA internalization by isolated rat jejunal enterocytes [80]. Exposing the cells to OEA stimulated the transcription of CD36 and, in parallel, increased the uptake of a fluorescently labeled FA [80]. This suggests that fatinduced OEA formation accelerates mucosal lipid absorption through an autocrine mechanism, an effect analogous to that produced by cholecystokinin via stimulation of pancreas and gall bladder secretion [81]. That gut OEA signaling might extend its functional reach beyond the control of food intake is further supported by two series of studies conducted independently by the laboratories of Angelo Izzo and Keith Sharkey. These investigators demonstrated that IP OEA injections delay gastric emptying and intestinal transit in mice through mechanism(s) that are independent of PPAR- $\alpha$ or TRPV1 activation [82-84]. The possible involvement of other lipid-sensing receptors, such as GPR119 or GPR120, deserves future investigation.

\section{Concluding remarks}

The evidence reviewed here indicates that the digestion of dietary fat triggers the mobilization of OEA in the epithelium of the upper small intestine, which initiates a cascade of molecular events that prolongs the interval between successive meals while accelerating FA uptake and slowing down gastrointestinal emptying (Figure 4). Because this combination of effects is expected to maximize lipid absorption, it is reasonable to hypothesize that a primary function of OEA signaling in the gut might be to mediate the adaptive response of this organ to the consumption of a large high-fat meal, an event that animals experience infrequently in the wild. In this context, the peculiar ability of OEA to enhance the consolidation of recent memories could also be rationalized as an adaptive strategy to improve foraging for essential, but relatively rare, fat-rich foods. Though 
triggered by dietary fat and strictly localized to the gut, OEA signaling is governed by the activity of the two main branches of the autonomic nervous system -sympathetic and parasympathetic - which appear to be the ultimate gatekeepers of this response.

As noted above, there are several questions that remain to be answered. Most pressing among them are those pertaining to the exact role of CD36 in OEA production, the nongenomic mechanisms engaged by OEA activation of PPAR- $\alpha$, and the identity of the receptor(s) involved in the regulatory actions of OEA on gastrointestinal motility. Despite these uncertainties, the hypothesis that OEA acts as a multifunctional fat sensor should provide a useful conceptual framework in which to address such questions and explore the possible involvement of this lipid mediator in obesity and other pathological conditions in which the physiological sensing of dietary fat might be imbalanced.

\section{Glossary}

\section{$\beta_{2}$-Adrenergic receptor}

Amygdala

Burmese python

CD36 (cluster of
differentiation 36)

Cholecystokinin

Consolidation

Endocannabinoids

Enterocytes

Glucagon-like peptide-1 (GLP-1) a $G$ protein-coupled receptor that recognizes the catecholamines, norepinephrine and epinephrine. It is linked to the activation of the enzyme adenylyl cyclase and the formation of the second messenger cAMP

a group of brain nuclei, also referred to as amygdaloid nuclear complex, located in the anteromedial temporal lobe. It is a key component of the limbic system (together with the olfactory cortex, the hippocampal formation, and the hypothalamus) and is involved in the control of emotion and drives

an ambush-eating snake species (Python molurus) that consumes huge meals after months of fasting and provides therefore a unique model of 'extreme' feeding behavior

also known as fatty acid transporter (FAT), CD36 is an integral membrane glycoprotein found on the cell surface of many cell types. It belongs to the class B scavenger receptor family and binds multiple ligands, including fatty acids and oxidized phospholipids

a gut peptide hormone secreted by endocrine I cells in the duodenum. It inhibits food intake and stimulates the release of digestive enzymes and bile from the pancreas and gallbladder, respectively. It is also present in the CNS where it acts as a neurotransmitter

the neural process through which short-term memories are gradually converted from into long-term memories

a family of endogenous lipid-derived agonists for $\mathrm{G}$ proteincoupled cannabinoid receptors, the molecular target of $\mathrm{D}^{9}$ tetrahydro-cannabinol in Cannabis. It includes anandamide and 2-arachidonoyl-sn-glycerol

the absorptive epithelial cells that line the lumen of the small intestine and colon

a gut peptide hormone secreted by endocrine L cells in the ileum. It suppresses glucagon secretion and causes glucosedependent stimulation of insulin secretion 


\section{Lamina propria (mucosae)}

\section{Leptin}

\section{Myenteric plexus (or Auerbach plexus)}

Nicotinic receptors

Nucleus of the solitary tract (NST)

Oxytocin

Plasmalogens

Stereospecific numbering (sn)

Vasopressin the layer of connective tissue underlying the epithelium of a mucous membrane

a protein hormone released by adipocytes and other cells. It is a key regulator of appetite, metabolism, and reproductive function

a portion of the enteric nervous system located in the muscular layer of the intestinal wall (muscularis propria). It controls the motility of the gastrointestinal tract

a group of ligand-gated ion channels activated by the neurotransmitter acetylcholine

a nucleus in the brainstem involved in the processing of taste information as well as in the regulation of cardiovascular, respiratory, and gastrointestinal functions

a neuropeptide secreted by neurons in the paraventricular and supraoptic nuclei of the hypothalamus, which plays important roles in the control of sexual reproduction, pair bonding, social recognition, and anxiety

naturally occurring glycerol-based lipids that contain an unsaturated ether moiety at the sn-1 position. Their functional significance is unknown

convention on how to designate the stereochemistry of glycerolbased lipids. When the glycerol is drawn in a Fisher projection with the secondary hydroxyl group to the left, the carbons are numbered 1,2,3 from top to bottom

a neuropeptide secreted by neurons in the paraventricular and supraoptic nuclei of the hypothalamus, and which plays important roles in the regulation of water retention and social behavior

\section{References}

1. Ackroff, K.; Sclafani, A. Oral and postoral determinants of dietary fat appetite. In: Montmayeur, JP.; le Coutre, J., editors. Fat Detection: Taste, Texture, and Post Ingestive Effects. CRC Press; 2010. p. 295-322.

2. Gaillard D, et al. The gustatory pathway is involved in CD36-mediated orosensory perception of long-chain fatty acids in the mouse. FASEB J. 2008; 22:1458-1468. [PubMed: 18162488]

3. Liang NC, et al. Sham feeding corn oil increases accumbens dopamine in the rat. Am J Physiol Regul Integr Comp Physiol. 2006; 291:R1236-R1239. [PubMed: 16763080]

4. DiPatrizio NV, et al. Endocannabinoid signal in the gut controls dietary fat intake. Proc Natl Acad Sci USA. 2011; 108:12904-12908. [PubMed: 21730161]

5. DiPatrizio NV, Piomelli D. The thrifty lipids: endocannabinoids and the neural control of energy conservation. Trends Neurosci. 2012; 20:1-9.

6. Fukuwatari T, et al. Expression of the putative membrane fatty acid transporter (FAT) in taste buds of the circumvallate papillae in rats. FEBS Lett. 1997; 414:461-464. [PubMed: 9315741]

7. Laugerette F, et al. CD36 involvement in orosensory detection of dietary lipids, spontaneous fat preference, and digestive secretions. J Clin Invest. 2005; 115:3177-3184. [PubMed: 16276419]

8. Degrace-Passilly P, Besnard P. CD36 and taste of fat. Curr Opin Clin Nutr Metab Care. 2012; 15:107-111. [PubMed: 22248592] 
9. Matsumura S, et al. GPR expression in the rat taste bud relating to fatty acid sensing. Biomed Res. 2007; 28:49-55. [PubMed: 17379957]

10. Cartoni C, et al. Taste preference for fatty acids is mediated by GPR40 and GP120. J Neurosci. 2010; 30:8376-8382. [PubMed: 20573884]

11. Pérez CA, et al. A transient receptor potential channel expressed in taste receptor cells. Nat Neurosci. 2002; 5:1169-1176. [PubMed: 12368808]

12. Liu P, et al. Transient receptor potential channel type M5 is essential for fat taste. J Neurosci. 2011; 31:8634-8642. [PubMed: 21653867]

13. Greenberg D, Smith GP. The controls of fat intake. Psychosom Med. 1996; 58:559-569. [PubMed: 8948004]

14. Sclafani A, et al. Selective effects of vagal deafferentation and celiac-superior mesenteric ganglionectomy on the reinforcing and satiating action of intestinal nutrients. Physiol Behav. 2003; 78:285-294. [PubMed: 12576127]

15. Randich A, et al. Responses of celiac and cervical vagal afferents to infusions of lipids in the jejunum or ileum of the rat. Am J Physiol Regul Integr Comp Physiol. 2000; 278:R34-R43. [PubMed: 10644619]

16. Zittel TT, et al. Fos protein expression in the nucleus of the solitary tract in response to intestinal nutrients in awake rats. Brain Res. 1994; 663:266-270. [PubMed: 7874510]

17. Schwartz GJ. Gut fat sensing in the negative feedback control of energy balance - recent advances. Physiol Behav. 2011; 104:621-623. [PubMed: 21557957]

18. Kuehl FA Jr, et al. The identification of $N$-(2-hydroxyethyl)-palmitamide as a naturally occurring anti-inflammatory agent. J Am Chem Soc. 1957; 79:5577-5578.

19. Bachur NR, et al. Fatty acid amides of ethanolamine in mammalian tissues. J Biol Chem. 1965; 240:1019-1024. [PubMed: 14284696]

20. Lambert DM, et al. The palmitoylethanolamide family: a new class of anti-Inflammatory agents? Curr Med Chem. 2002; 9:663-674. [PubMed: 11945130]

21. Devane WA, et al. Isolation and structure of a brain constituent that binds to the cannabinoid receptor. Science. 1992; 258:1946-1949. [PubMed: 1470919]

22. Piomelli D. The molecular logic of endocannabinoid signaling. Nat Rev Neurosci. 2003; 4:873884. [PubMed: 14595399]

23. Rodriguez de Fonseca F, et al. An anorexic lipid mediator regulated by feeding. Nature. 2001; 414:209-212. [PubMed: 11700558]

24. Fu J, et al. Oleylethanolamide regulates feeding and body weight through activation of the nuclear receptor PPAR-a. Nature. 2003; 425:90-93. [PubMed: 12955147]

25. Calignano A, et al. Control of pain initiation by endogenous cannabinoids. Nature. 1998; 394:277281. [PubMed: 9685157]

26. LoVerme J, et al. Rapid broad-spectrum analgesia through activation of peroxisome proliferationactivated receptor-alpha. J Pharmacol Exp Ther. 2006; 319:1051-1061. [PubMed: 16997973]

27. Sasso O, et al. Antinociceptive effects of the $N$-acylethanolamine acid amidase inhibitor ARN077 in rodent pain models. Pain. 2013; 154:350-360. [PubMed: 23218523]

28. Lo Verme J, et al. The nuclear receptor peroxisome proliferator-activated receptor-alpha mediates the anti-inflammatory actions of palmitoylethanolamide. Mol Pharmacol. 2005; 67:15-19. [PubMed: 15465922]

29. Solorzano C, et al. Selective $N$-acylethanolamine-hydrolyzing acid amidase inhibition reveals a key role for endogenous palmitoylethanolamide in inflammation. Proc Natl Acad Sci USA. 2009; 106:20966-20971. [PubMed: 19926854]

30. Melis M, et al. Endogenous fatty acid ethanolamides suppress nicotine-induced activation of mesolimbic dopamine neurons through nuclear receptors. J Neurosci. 2008; 28:13985-13994. [PubMed: 19091987]

31. Schmid $\mathrm{HH}$, et al. The $N$-acylation-phosphodiesterase patway and cell signaling. Chem Phys Lipids. 1996; 80:133-142. [PubMed: 8681424]

32. Cadas $\mathrm{H}$, et al. Occurrence and biosynthesis of edogenous cannabinoid precursor, $N$-arachidonoyl phosphatidylethanolamine, in rat brain. J Neurosci. 1997; 17:1226-1242. [PubMed: 9006968] 
33. Fu J, et al. Food intake regulates oleoylethanolamide formation and degradation in the proximal small intestine. J Biol Chem. 2007; 282:1518-1528. [PubMed: 17121838]

34. Astarita G, et al. Identification of biosynthetic precursors for the endocannabinoid anandamide in the rat brain. J Lipid Res. 2008; 49:48-57. [PubMed: 17957091]

35. Okamoto Y, et al. Molecular characterization of a phospholipase D generating anandamide and its congeners. J Biol Chem. 2004; 279:5298-5305. [PubMed: 14634025]

36. Cravatt BF, Simon GM. Characterization of mice lacking candidate $\mathrm{N}$-acyl ethanolamine biosynthetic enzymes provides evidence for multiple pathways that contribute to endocannabinoid production in vivo. Mol Biosyst. 2010; 6:1411-1418. [PubMed: 20393650]

37. Tsuboi K, et al. Enzymatic formation of $N$-acylethanolamines from $N$-acylethanolamine plasmalogen through $\mathrm{N}$-acylphosphatidylethanolamine-hydrolyzing phospholipase D-dependent and -independent pathways. Biochim Biophys Acta. 2011; 1811:565-577. [PubMed: 21801852]

38. Cravatt BF, et al. Molecular characterization of an enzyme that degrades neuromodulatory fattyacid amides. Nature. 1996; 384:83-87. [PubMed: 8900284]

39. Tsuboi K, et al. Molecular characterization of $N$-acylethanolamine-hydrolyzing acid amidase, a novel member of the choloylglycine hydrolase family with structural and functional similarity to acid ceramidase. J Biol Chem. 2005; 280:11082-11092. [PubMed: 15655246]

40. Katayama K, et al. Distribution of anandamide amidohydrolase in rat tissues with special reference to small intestine. Biochim Biophys Acta. 1997; 1347:2012-2018.

41. Gulyas AI, et al. Segregation of two endocannabinoid-hydrolyzing enzymes into pre and postsynaptic compartments in the rat hippocampus, cerebellum and amygdala. Eur J Neurosci. 2004; 20:441-458. [PubMed: 15233753]

42. Wei BQ, et al. A second fatty acid amide hydrolase with variable distribution among placental mammals. J Biol Chem. 2006; 281:36569-36578. [PubMed: 17015445]

43. Tsuboi K, et al. The $N$-acylethanolamine-hydrolyzing acid amidase (NAAA). Chem Biodivers. 2007; 4:1914-1925. [PubMed: 17712833]

44. Petersen $\mathrm{G}$, et al. Intestinal levels of anandamide and oleoylethanolamide in food-deprived rats are regulated through their precursors. Biochim Biophys Acta. 2006; 1761:143-150. [PubMed: 16478679]

45. Astarita G, et al. Postprandial increase of oleoylethanolamide mobilization in small intestine of the Burmese python (Python molurus). Am J Physiol Regul Integr Comp Physiol. 2006; 290:R1407R1412. [PubMed: 16373434]

46. Schwartz GJ, et al. The lipid messenger OEA links dietary fat intake to satiety. Cell Metab. 2008; 8:281-288. [PubMed: 18840358]

47. Guijarro A, et al. CD36 gene deletion decreases oleoylethanolamide levels in small intestine of free-feeding mice. Pharmacol Res. 2010; 61:27-33. [PubMed: 19778614]

48. Poirier $\mathrm{H}$, et al. Localization and regulation of the putative membrane fatty-acid transporter (FAT) in the small intestine. Comparison with fatty acid-binding proteins (FABP). Eur J Biochem. 1996; 238:368-373. [PubMed: 8681947]

49. Huang MM, et al. MembraneglycoproteinIV(CD36)isphysically associated with the Fyn, Lyn and Yes protein-tyrosine kinases in human platelets. Proc Natl Acad Sci USA. 1991; 88:7844-7848. [PubMed: 1715582]

50. Naville D, et al. Link between intestinal CD36 ligand binding and satiety induced by a high protein diet in mice. PLoS ONE. 2012; 7:E30686. [PubMed: 22295104]

51. Nassir F, et al. CD36 is important for fatty acid and cholesterol uptake by the proximal but not distal intestine. J Biol Chem. 2007; 282:19493-19501. [PubMed: 17507371]

52. Artmann A, et al. Influence of dietary fatty acids on endocannabinoid and $N$-acylethanolamine levels in rat brain, liver and small intestine. Biochim Biophys Acta. 2008; 1781:200-212. [PubMed: 18316044]

53. Diep TA, et al. Dietary fat decreases intestinal levels of the anorectic lipids through a fat sensor. FASEB J. 2011; 25:765-774. [PubMed: 20959516]

54. Gaetani S, et al. Modulation of meal pattern in the rat by the anorexic lipid mediator oleoylethanolamide. Neuropsychopharmacology. 2003; 28:1311-1316. [PubMed: 12700681] 
55. Oveisi F, et al. Oleoylethanolamide inhibits food intake in free-feeding rats after oral administration. Pharmacol Res. 2004; 49:461-466. [PubMed: 14998556]

56. Nielsen MJ, et al. Food intake is inhibited by oral oleoylethanolamide. J Lipid Res. 2004; 45:10271029. [PubMed: 15060091]

57. Proulx K, et al. Mechanism of oleoylethanolamide-induced changes in feeding behavior and motor activity. Am J Physiol Regul Integr Comp Physiol. 2005; 289:R729-R737. [PubMed: 15879057]

58. Astarita G, et al. Pharmacological characterization of hydrolysis-resistant analogs of oleoylethanolamide with potent anorexiant properties. J Pharmacol Exp Ther. 2006; 318:563-570. [PubMed: 16702440]

59. Ritter RC, et al. Cholecystokinin: proofs and prospects for involvement in control of food intake and body weight. Neuropeptides. 1999; 33:387-399. [PubMed: 10657516]

60. Clifton PG, et al. Similarities in the action of Ro 60-0175, a 5- $\mathrm{HT}_{2 \mathrm{C}}$ receptor agonist and dfenfluramine on feeding patterns in the rat. Psychopharmacology (Berl). 2000; 152:256-267. [PubMed: 11105935]

61. Fu J, et al. Targeted enhancement of oleoylethanolamide production in proximal small intestine induces across-meal satiety in rats. Am J Physiol Regul Integr Comp Physiol. 2008; 295:R45R50. [PubMed: 18434444]

62. Pawar A, Jump DB. Unsaturated fatty acid regulation of peroxisome proliferatior-activated receptor alpha activity in rat primary hepatocytes. J Biol Chem. 2003; 278:35931-35939. [PubMed: 12853447]

63. Chakravarthy MV, et al. 'New' hepatic fat activates PPAR-a to maintain glucose, lipid, and cholesterol homeostasis. Cell Metab. 2005; 1:309-322. [PubMed: 16054078]

64. Khasabova IA, et al. Peroxisome proliferator-activated receptor a mediates acute effects of palmitoylethanolamide on sensory neurons. J Neurosci. 2012; 32:12735-12743. [PubMed: 22972997]

65. Melis M, et al. Peroxisome proliferator-activated receptors-alpha modulate dopamine cell activity through nicotinic receptors. Biol Psychiatry. 2010; 68:256-264. [PubMed: 20570248]

66. Ahern GP. Activation of TRPV1 by the satiety factor oleoylethanolamide. J Biol Chem. 2003; 278:30429-30434. [PubMed: 12761211]

67. Wang X, et al. Oleoylethanolamide excites vagal sensory neurons, induces visceral pain and reduces short-term food intake in mice via capsaicin receptor TRPV1. J Physiol. 2005; 564:541547. [PubMed: 15695242]

68. Overton HA, et al. Deorphanization of a G protein-coupled receptor for oleoylethanolamide and its use in the discovery of small-molecule hypophagic agents. Cell Metab. 2006; 3:167-175. [PubMed: 16517404]

69. Hansen HS, et al. GPR119 as a fat sensor. Trends Pharmacol Sci. 2012; 33:374-381. [PubMed: 22560300]

70. Lauffer LM, et al. GPR119 is essential for oleoylethanolamide-induced glucagon-like peptide-1 secretion from the intestinal enteroendocrine L-cell. Diabetes. 2009; 58:1058-1066. [PubMed: 19208912]

71. LoVerme J, et al. Regulation of food intake by oleoylethanolamide. Cell Mol Life Sci. 2005; 62:708-716. [PubMed: 15770421]

72. Lan H, et al. GPR119 is required for physiological regulation of glucagon-like peptide-1 secretion but not for metabolic homeostasis. J Endocrinol. 2009; 201:219-230. [PubMed: 19282326]

73. Berthoud HR, Morrison C. The brain, appetite, and obesity. Annu Rev Psychol. 2008; 59:55-92. [PubMed: 18154499]

74. Gaetani S, et al. The fat-induced satiety factor oleoylethanolamide suppresses feeding through central release of oxytocin. J Neurosci. 2010; 30:8096-8101. [PubMed: 20554860]

75. Fu J, et al. Sympathetic activity controls fat-induced oleoylethanolamide signaling in small intestine. J Neurosci. 2011; 31:5730-5736. [PubMed: 21490214]

76. Lomax AE, et al. The participation of the sympathetic innervation of the gastrointestinal tract in disease states. Neurogastroenterol Motil. 2010; 22:7-18. [PubMed: 19686308] 
77. Insel TR, Young JL. The neurobiology of attachment. Nat Rev Neurosci. 2001; 2:129-136. [PubMed: 11252992]

78. McGaugh JL. Memory - a century of consolidation. Science. 2000; 287:248-251. [PubMed: 10634773]

79. Campolongo P, et al. Fat-induced satiety factor oleoylethanolamide enhances memory consolidation. Proc Natl Acad Sci USA. 2009; 106:8027-8031. [PubMed: 19416833]

80. Yang Y, et al. Mechanism of oleoylethanolamide on fatty acid uptake in small intestine after food intake and body weight reduction. Am J Physiol Regul Integr Comp Physiol. 2007; 292:R235R241. [PubMed: 16902188]

81. Dockray GJ. Cholecystokinin. Curr Opin Endocrinol Diabetes Obes. 2012; 19:8-12. [PubMed: 22157397]

82. Aviello G, et al. Inhibitory effect of the anorexic compound oleoylethanolamide in gastric emptying in control and overweight mice. J Mol Med. 2008; 86:413-422. [PubMed: 18278475]

83. Cluny NL, et al. The identification of peroxisome proliferatior-activated receptor alphaindependent effects of oleoylethanolamide on intestinal transit in mice. Neurogastroenterol Motil. 2009; 21:420-429. [PubMed: 19140957]

84. Borrelli F, Izzo AA. Role of acylethanolamides in the gastrointestinal tract with special reference to food intake and energy balance. Best Pract Res Clin Endocrinol Metab. 2009; 23:33-49. [PubMed: 19285259]

85. Kilaru A, et al. The $\mathrm{N}$-Acylethanolamine-mediated regulatory pathway in plants. Chem Biodivers. 2007; 4:1933-1955. [PubMed: 17712835]

86. Scuderi C, et al. Palmitoylethanolamide exerts neuroprotective effects in mixed neuroglial cultures and organotypic hippocampal slices via peroxisome proliferator-activated receptor-a. $\mathbf{J}$ Neuroinflammation. 2012; 9:49. [PubMed: 22405189]

87. Zhu C, et al. Proinflammatory stimuli control $\mathrm{N}$-acylphosphatidylethanolamine-specific phospholipase D expression in macrophages. Mol Pharmacol. 2011; 79:786-792. [PubMed: 21233218]

88. Gillum MP, et al. $N$-acylphosphatidylethanolamine, a gut-derived circulating factor induced by fat ingestion, inhibits food intake. Cell. 2008; 135:813-824. [PubMed: 19041747]

89. Wellner N, et al. Studies on the anorectic effect of $N$-acylphosphatidylethanolamine and phosphatidylethanolamine in mice. Biochim Biophys Acta. 2011; 1811:508-512. [PubMed: 21723414]

90. LoVerme J, et al. Cold exposure stimulates synthesis of the bioactive lipid oleoylethanolamide in rat adipose tissue. J Biol Chem. 2006; 281:22815-22818. [PubMed: 16785227]

91. Guzmán M, et al. Oleoylethanolamide stimulates lipolysis by activating the nuclear receptor peroxisome proliferator-activated receptor-a (PPAR-a). J Biol Chem. 2004; 279:27849-27854. [PubMed: 15123613]

92. Thabuis $\mathrm{C}$, et al. Analysis of gene expression pattern reveals potential targets of dietary oleoylethanolamide in reducing body fat gain in C3H mice. J Nutr Biochem. 2010; 21:922-928. [PubMed: 19954948]

93. Thabuis C, et al. Lipid transport function is the main target of oral oleoylethanolamide to reduce adiposity in high-fat-fed mice. J Lipid Res. 2011; 52:1373-1382. [PubMed: 21515921] 


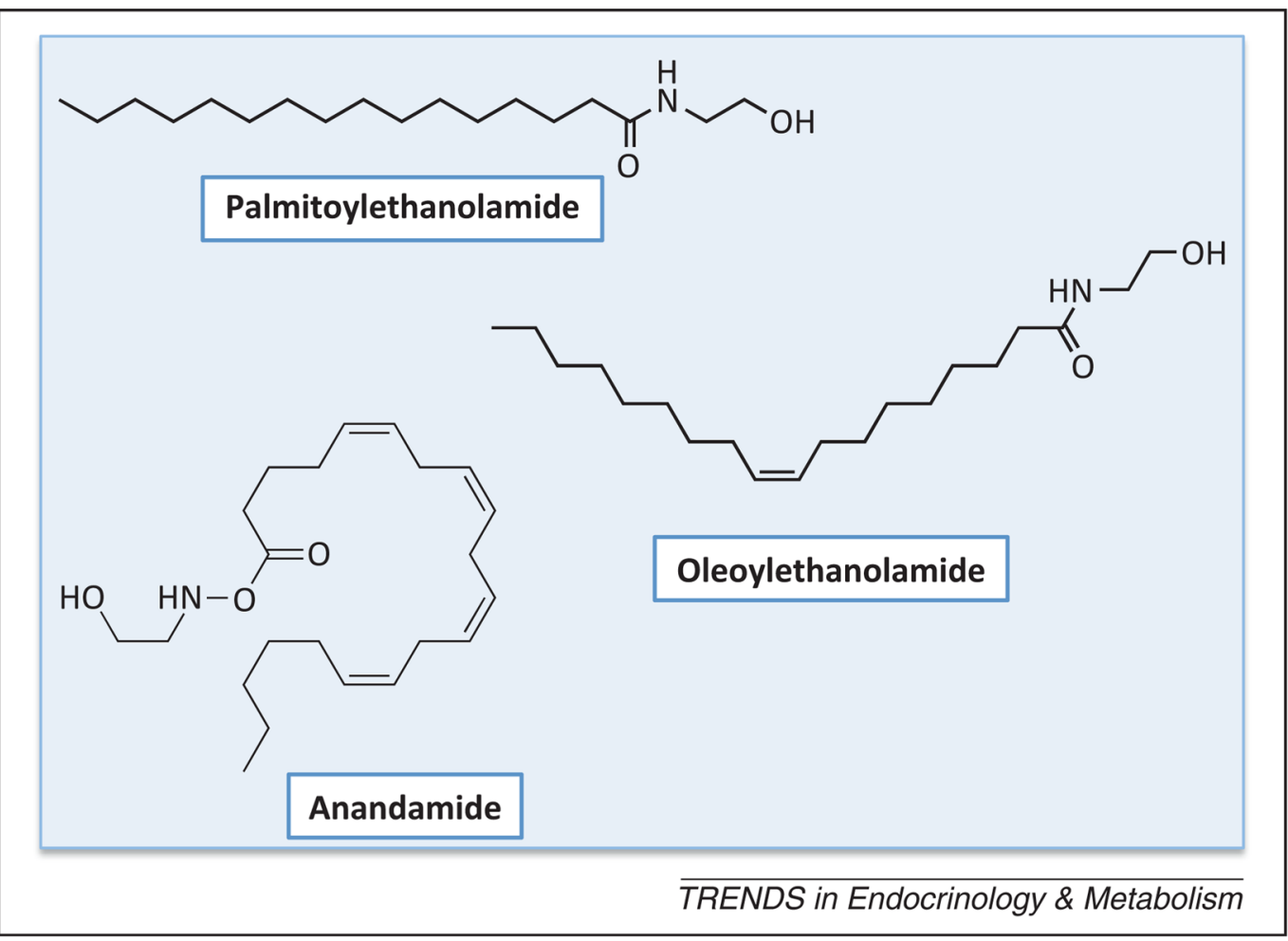

Figure 1.

Chemical structures of three representative fatty acid ethanolamides (FAEs).

Palmitoylethanolamide, the first FAE to be discovered, and oleoylethanolamide exert most, albeit not all, of their biological effects by engaging peroxisome proliferator-activated receptor- $\alpha$ (PPAR- $a)$, a ligand-activated transcription factor that can also trigger nongenomic signaling events. Anandamide (arachidonoylethanolamide) is an endogenous agonist for $\mathrm{G}$ protein-coupled cannabinoid receptors. 


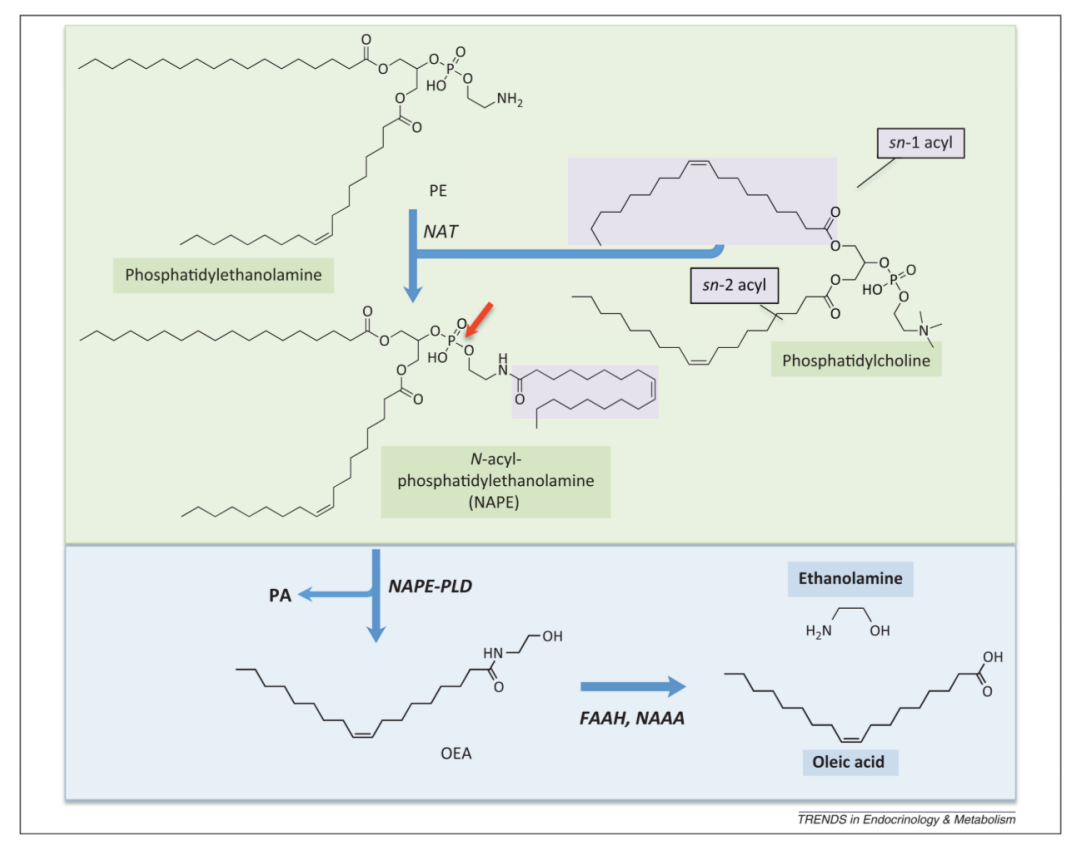

Figure 2.

Formation and degradation of oleoylethanolamide (OEA) in mammalian cells. A calciumdependent $N$-acyltransferase activity (NAT) catalyzes the transfer of a fatty acyl group from the $s n-1$ position of phosphatidylcholine to the amine group of phosphatidylethanolamine, producing a family of $N$-acylphosphatidylethanolamine (NAPE) species. NAPEs containing oleic acid at the amine position (shown in figure) generate OEA upon hydrolysis of their distal phosphodiester bond (red arrow). This reaction is catalyzed by a NAPE-selective phospholipase D (NAPE-PLD), which produces phosphatidic acid (PA) as a byproduct. OEA is hydrolyzed into oleic acid and ethanolamine by either of two structurally unrelated enzymes: FA amide hydrolase (FAAH) or $N$-acylethanolamine acid amidase (NAAA). 


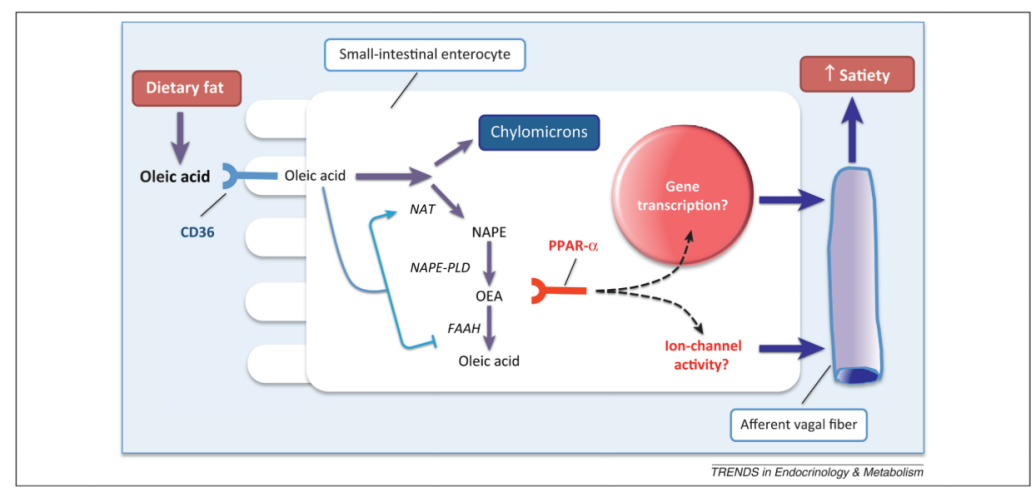

Figure 3.

Oleoylethanolamide (OEA) signaling as a biosensor for dietary fat. According to the model shown in this figure, fat digestion in the small-intestinal lumen releases free oleic acid, which is internalized by the enterocytes and funneled either toward the formation of chylomicrons, or toward the production of OEA through the NAT/NAPE-PLD pathway (see Figure 2). The latter process requires CD36, but the exact roles of this cell-membrane protein are unclear. In addition to enabling oleic acid transport, CD36 might regulate the activities of NAPE-PLD and fatty acid amide hydrolase (FAAH), possibly by recruiting intracellular signal transduction pathways (symbolized by the arrows). Newly formed OEA binds to PPAR-a, which activates sensory fibers of the vagus nerve through an as-yet undefined mechanism, leading to the enhancement of satiety. 


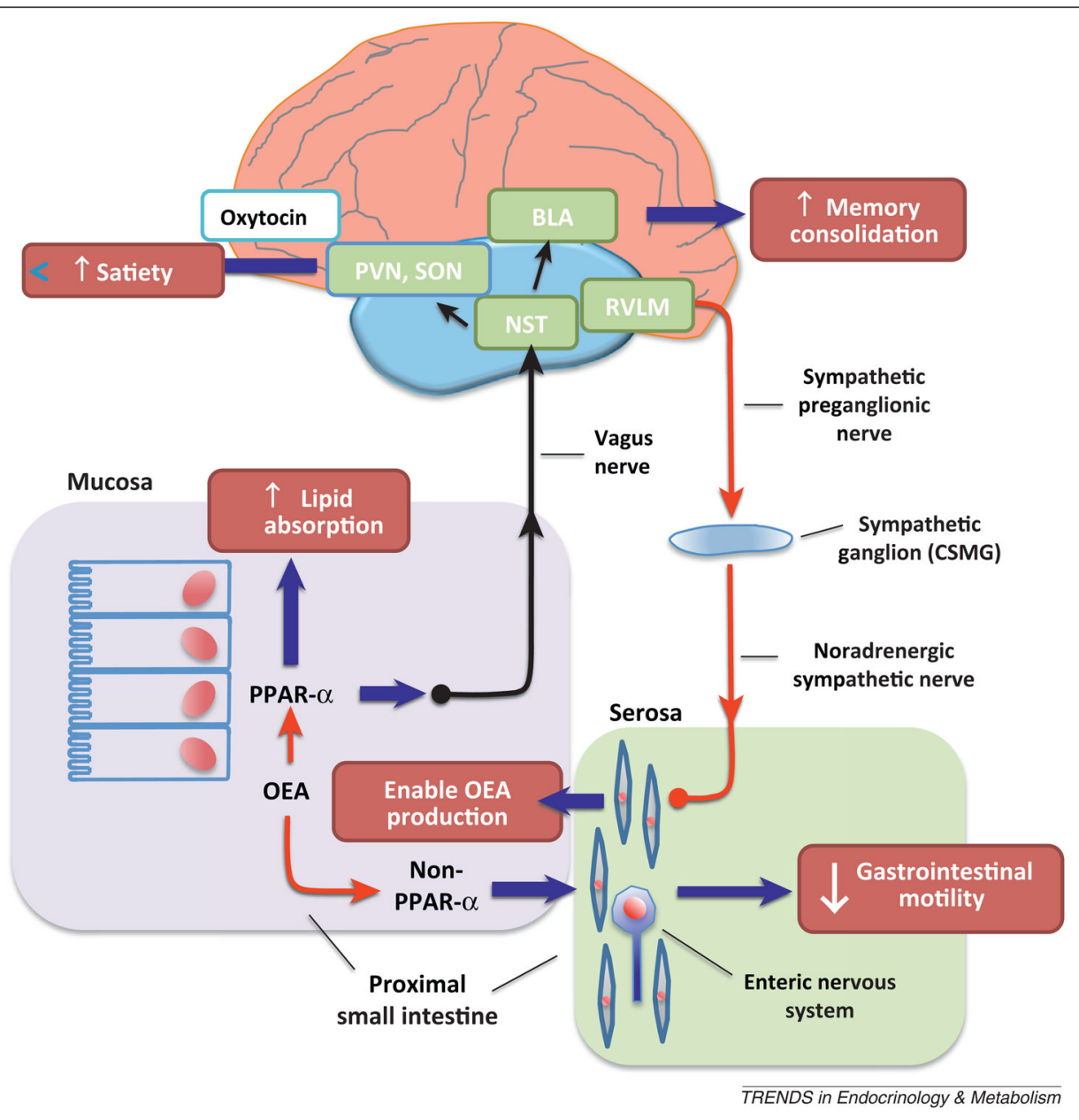

Figure 4.

From gut to brain and back. The entry of oleic acid into gut mucosal cells stimulates oleoylethanolamide (OEA) mobilization, which launches a PPAR-a-mediated signal that travels to the CNS through the afferent vagus nerve. From the brainstem nucleus of the solitary tract (NST), the signal is relayed to magnocellular neurons of the paraventricular (PVN) and supraoptic (SON) nuclei of the hypothalamus, stimulating oxytocin neurotransmission. This neural peptide, whose ability to control satiety is most likely mediated by activation of descending projections from hypothalamus to brainstem, plays an obligatory role in OEA-induced satiety. The PPAR-a-mediated signal may also travel to the basolateral nucleus of the amygdala (BLA) to strengthen the consolidation of recently formed memories. Within the gut, OEA may influence fatty acid (FA) absorption (through PPAR- $\boldsymbol{\alpha}$ ) as well as smooth-muscle motility (through an as-yet unidentified receptor). A descending sympathetic pathway, presumably originating in the rostral ventrolateral medulla (RVLM), facilitates OEA production by activating $\beta$-adrenergic receptors in the submucosal layer and the myenteric plexus. 